\title{
Cubication of conservative nonlinear oscillators
}

\author{
Augusto Beléndez ${ }^{(1)}$, Mariela L. Álvarez ${ }^{(1)}$, Elena Fernández ${ }^{(2)}$ and \\ Inmaculada Pascual ${ }^{(2)}$
}

(1) Departamento de Física, Ingeniería de Sistemas y Teoría de la Señal.

Universidad de Alicante. Apartado 99. E-03080 Alicante. SPAIN

(2) Departamento de Óptica, Farmacología y Anatomía.

Universidad de Alicante. Apartado 99. E-03080 Alicante. SPAIN

Corresponding author: A. Beléndez (E-mail: a.belendez@ua.es, Phone:+34965903651, Fax: +34965903464)

\begin{abstract}
A cubication procedure of the nonlinear differential equation for conservative nonlinear oscillators is analyzed and discussed. This scheme is based on the Chebyshev series expansion of the restoring force and this allows us to approximate the original nonlinear differential equation by a Duffing equation in which the coefficients for the linear and cubic terms depend on the initial amplitude, $A$, while in a Taylor expansion of the restoring force these coefficients are independent of $A$. The replacement of the original nonlinear equation by an approximate Duffing equation allows us to obtain an approximate frequencyamplitude relation as a function of the complete elliptic integral of the first kind. Some conservative nonlinear oscillators are analyzed to illustrate the usefulness and effectiveness of this scheme.
\end{abstract}

Keywords: Nonlinear oscillators; Approximate solutions; Chebyshev polynomials. 
Beléndez, A.; Álvarez, M. L., Fernández, E., Pascual, I., "Cubication of conservative nonlinear oscillators", European Journal of Physics, Vol. 30, № 5, pp. 973-981 (2009). doi:10.1088/0143-0807/30/5/006

\section{Introduction}

In a previous paper [1] we have shown that it is possible to replace the second-order nonlinear differential equation which governs the behaviour of a conservative nonlinear oscillator with a linear equation that approximates the original nonlinear equation closely enough to give accurate results. This linearization scheme uses the Chebyshev series expansion of the restoring force is used instead of the Taylor one [2-4] and an approximate frequency depending on the oscillation amplitude is obtained. In this paper we present a generalization of this technique in which the original second-order nonlinear differential equation is replaced with the well-known Duffing equation, and this cubic equation is exactly solved. To do this approximation the Chebychev series expansion of the restoring force is also used instead of the Taylor one [2,3]. Some examples of conservative nonlinear oscillators are analyzed and we can see the approximate frequencies obtained are valid not only for small but also for large values of the oscillation amplitude and, in some situations, these approximate frequencies are valid for the complete range of oscillation amplitude, including the limiting cases of amplitude approaching zero and infinity. Excellent agreement of the approximate frequencies with the exact ones will be demonstrated and discussed. We think this study can be interesting for students of Classical Mechanics courses at university level.

\section{Formulation and solution method}

Consider the second order differential equation, which governs the motion of a nonlinear conservative oscillator

$$
\frac{\mathrm{d}^{2} x}{\mathrm{~d} t^{2}}+f(x)=0
$$

with initial conditions

$$
x(0)=A, \quad \frac{\mathrm{d} x}{\mathrm{~d} t}(0)=0
$$

We suppose that the nonlinear function $f(x)$ is odd, i.e. $f(-x)=-f(-x)$ and satisfies $x f(x)>0$ for $x \in[-A, A], x \neq 0, A$ being the amplitude of motion. The motion is assumed to be periodic 
and the problem is to determine the angular frequency of oscillation and corresponding solution as a function of the system parameters and the amplitude $A$.

In order to derive simple analytical formulae we can expand the function $f(x)$ in Taylor series around the equilibrium point $x=0$ and the following equation is obtained

$$
f(x)=\sum_{n=0}^{\infty} \frac{f^{(2 n+1)}(0)}{(2 n+1) !} x^{2 n+1}=\sum_{n=0}^{\infty} a_{2 n+1} x^{2 n+1}
$$

where

$$
a_{2 n+1}=\frac{1}{(2 n+1) !}\left(\frac{\mathrm{d}^{2 n+1} f(x)}{\mathrm{d} x^{2 n+1}}\right)_{x=0}
$$

and we have taken into account that the function $f(x)$ is odd and then the series expansion contains only terms involving odd powers of $x$. For small values of $x$ (and then of $A$ ) it is possible to do the following cubic approximation

$$
f(x)=a_{1} x+a_{3} x^{3}
$$

From Eq. (4), $a_{1}$ and $a_{3}$ do not depend on the oscillation amplitude $A$. Substituting Eq. (5) into Eq. (1) we obtain the well-known liner differential equation for the Duffing oscillator [5-7]

$$
\frac{\mathrm{d}^{2} x}{\mathrm{~d} t^{2}}+a_{1} x+a_{3} x^{3}=0
$$

whose frequency and solution are given as follows [6,7]

$$
\begin{gathered}
\omega_{T}(A)=\frac{\pi \sqrt{a_{1}+a_{3} A^{2}}}{2 K(m)}, \quad m=\frac{a_{3} A^{2}}{2\left(a_{1}+a_{3} A^{2}\right)} \\
x_{T}(t)=A \operatorname{cn}\left[t \sqrt{a_{1}+a_{3} A^{2}} ; m\right]
\end{gathered}
$$


Beléndez, A.; Álvarez, M. L., Fernández, E., Pascual, I., "Cubication of conservative nonlinear oscillators", European Journal of Physics, Vol. 30, № 5, pp. 973-981 (2009). doi:10.1088/0143-0807/30/5/006

where $\operatorname{cn}\left[t \sqrt{a_{1}+a_{3} A^{2}} ; m\right]$ is the Jacobi elliptic function and $K(m)$ is the complete elliptic integral of the first kind defined as follows

$$
K(m)=\int_{0}^{\pi / 2} \frac{\mathrm{d} \theta}{\sqrt{1-m \sin ^{2} \theta}}
$$

However, the results obtained with this approximation will be only valid for small amplitudes and only when the series expansion in Eq. (2) can be carried out (see the discussion of this fact in references [1] and [8]).

In order to approximately solve the Eq. (1) by means a better cubication procedure, a reduced variable $y=x / A$ is introduced in Eqs. (1) and (2)

$$
\begin{aligned}
& \frac{\mathrm{d}^{2} y}{\mathrm{~d} t^{2}}+\frac{1}{A} f(A y)=0 \\
& y(0)=1, \quad \frac{\mathrm{d} y}{\mathrm{~d} t}(0)=0
\end{aligned}
$$

Following Denman [2] and Jonckheere [3], the function $f(A y)$ is expanding in terms of Chebyshev polynomials of the first kind $T_{n}(x)$ as follows [2,3]

$$
f(A y)=\sum_{n=0}^{\infty} b_{2 n+1}(A) T_{2 n+1}(y)
$$

where the first two polynomials are $[9,10]$

$$
T_{1}(y)=y, \quad T_{3}(y)=4 y^{3}-3 y,
$$

and $[9,10]$ 
Beléndez, A.; Álvarez, M. L., Fernández, E., Pascual, I., "Cubication of conservative nonlinear oscillators", European Journal of Physics, Vol. 30, № 5, pp. 973-981 (2009). doi:10.1088/0143-0807/30/5/006

$$
b_{2 n+1}(A)=\frac{2}{\pi} \int_{-1}^{+1}\left(1-y^{2}\right)^{-1 / 2} f(A y) T_{2 n+1}(y) \mathrm{d} y
$$

which are amplitude dependent. In Eq. (14) we have taken into account that $f(x)$ is an odd function of $x$. We can approximate Eq. (12) retaining only the first two terms as follows

$$
f(A y) \approx b_{1}(A) T_{1}(y)+b_{3}(A) T_{3}(y)=\left[b_{1}(A)-3 b_{3}(A)\right] y+4 b_{3}(A) y^{3}=\frac{b_{1}(A)-3 b_{3}(A)}{A} x+\frac{4 b_{3}(A)}{A^{3}} x^{3}
$$

The nonlinear differential equation in (1) can be then approximated by the following Duffing equation

$$
\frac{\mathrm{d}^{2} x}{\mathrm{~d} t^{2}}+\alpha(A) x+\beta(A) x^{3}=0
$$

where

$$
\alpha(A)=\frac{b_{1}(A)-3 b_{3}(A)}{A}
$$

and

$$
\beta(A)=\frac{4 b_{3}(A)}{A^{3}}
$$

Then, approximate frequency and solution for the initial equation will be exact frequency and solution for the Duffing equation, and it will give as follows

$$
\begin{gathered}
\omega_{C h}(A)=\frac{\pi \sqrt{\alpha+\beta A^{2}}}{2 K(m)}, \quad m=\frac{\beta A^{2}}{2\left(\alpha+\beta A^{2}\right)} \\
x_{C h}(t)=A \operatorname{cn}\left[t \sqrt{\alpha+\beta A^{2}} ; m\right]
\end{gathered}
$$


Substituting Eqs. (17) and (18) into Eq. (19) we can write the approximate frequency as a function of the first two terms of the Chebyshev series expansion of the nonlinear function $f(x)$ as follows

$$
\omega_{C h}(A)=\frac{\pi \sqrt{b_{1}+b_{3}}}{2 \sqrt{A} K\left(\frac{2 b_{3}}{b_{1}+b_{3}}\right)}
$$

where $b_{1}$ and $b_{3}$ depend on the initial amplitude $A$ (see Eq. (14)).

\section{Examples}

In this section, we present some examples to illustrate the usefulness and effectiveness of the cubication technique based on the Chebyshev series expansion.

\section{1.- The simple pendulum}

This is perhaps one of the systems more times analyzed approximately [11-16]. The nonlinear differential equation for the simple pendulum is

$$
\frac{\mathrm{d}^{2} x}{\mathrm{~d} t^{2}}+\omega_{0}^{2} \sin x=0
$$

with initial conditions given in Eq. (2). From Eq. (14) we obtain

$$
b_{1}=2 \omega_{0}^{2} J_{1}(A), \quad b_{3}=-2 \omega_{0}^{2} J_{3}(A)
$$

where $J_{1}(A)$ and $J_{3}(A)$ are the first-order and third-order Bessel functions of the first kind, respectively. Substituting in Eq. (21), the approximate frequency can be written as follows

$$
\omega_{C h}(A)=\frac{\pi \omega_{0} \sqrt{J_{1}(A)-J_{3}(A)}}{2 \sqrt{A} K\left(\frac{2 J_{3}(A)}{J_{3}(A)-J_{1}(A)}\right)}
$$


In Figure 1 we compare the nonlinear function $f(x) / \omega_{0}^{2}=\sin x$ with its cubic approximations obtained using the Taylor series expansion, $f_{T}(x) / \omega_{0}^{2}=x-\frac{1}{6} x^{3}$, and the Chebyshev series expansion $f_{C h}(x) / \omega_{0}^{2}=\left(2 J_{1}(A)+6 J_{3}(A)\right)(x / A)-8 J_{3}(A)(x / A)^{3}$.

For the simple pendulum the exact frequency $\omega_{e}(A)$ is given as follows [1]

$$
\omega_{e}(A)=\frac{\pi \omega_{0}}{2 K\left(\sin ^{2} \frac{A}{2}\right)}
$$

It is easy to verify that the relative errors of the approximate frequencies $\omega_{T}(A)$ (using the Taylor expansion we obtain $a_{1}=\omega_{0}^{2}$ and $\left.a_{3}=-\frac{1}{6} \omega_{0}^{2}\right)$ and $\omega_{C h}(A)$ are less than $1 \%$ for $A<45^{\circ}$ and $A<155^{\circ}$, respectively.

\section{2.- Antisymmetric, constant force oscillator}

Suppose that a particle of mass $m$ moving in one dimension, $x$, has a state of stable equilibrium produced by a force that is directed toward the origin and has a constant magnitude $F(x)=-F_{0} \operatorname{sgn}(x)$ where $F_{0}$ is a positive constant and

$$
\operatorname{sgn}(x)=\left\{\begin{array}{cc}
1, & x>0 \\
0, & x=0 \\
-1, & x<0
\end{array}\right.
$$

The nonlinear differential equation for this case is

$$
\frac{\mathrm{d}^{2} x}{\mathrm{~d} t^{2}}+\lambda^{2} \operatorname{sgn}(x)=0, \quad \lambda=\sqrt{\frac{F_{0}}{m}}
$$

with initial conditions given in Eq. (2). 
Beléndez, A.; Álvarez, M. L., Fernández, E., Pascual, I., "Cubication of conservative nonlinear oscillators", European Journal of Physics, Vol. 30, № 5, pp. 973-981 (2009). doi:10.1088/0143-0807/30/5/006

The oscillations corresponding to Eq. (27) are intrinsically nonlinear and the function $\lambda^{2} \operatorname{sgn}(x)$ cannot be expanded in Taylor series. However, we can use the Chebyshev series expansion and we obtain

$$
b_{1}=\frac{4 \lambda^{2}}{\pi}, \quad b_{3}=-\frac{4 \lambda^{2}}{3 \pi}
$$

which allows us to write the approximately frequency for this oscillator as follows

$$
\omega_{C h}(A)=\frac{2 \lambda}{K(1 / 2)} \sqrt{\frac{\pi}{3 A}} \approx \frac{1.103878 \lambda}{A}
$$

In Figure 2 we compare the nonlinear function $f(x) / \lambda^{2}=\operatorname{sgn}(x)$ with its cubic approximation obtained using the Chebyshev series expansion, $f_{C h}(x) / \lambda^{2}=\frac{8}{\pi A} x-\frac{16}{\pi A^{3}} x^{3}$.

Direct integration of Eq. (26) yields the exact frequency $\omega_{e}(A)$ as follows [6]

$$
\omega_{e}(A)=\frac{\pi \lambda}{2 \sqrt{2 A}}
$$

We can easily verify that

$$
\frac{\omega_{C h}(A)}{\omega_{e}(A)}=\frac{4 \sqrt{2}}{\sqrt{3 \pi} K(1 / 2)} \approx 0.993830
$$

This means that the relative error of the approximate frequency is less than $0.62 \%$ for all oscillation amplitudes.

\section{5.- Mass attached to two stretched elastic springs}

The nonlinear differential equation for this case can be written as follows $[1,5,6,17]$

$$
\frac{\mathrm{d}^{2} x}{\mathrm{~d} t^{2}}+\omega_{0}^{2}\left(1-\frac{\lambda}{\sqrt{1+x^{2}}}\right) x=0
$$


where $0<\lambda \leq 1$. From Eq. (14) we obtain

$$
\begin{gathered}
b_{1}=\omega_{0}^{2} A\left[1-\frac{4 \lambda}{\pi A^{2}}\left(\sqrt{1+A^{2}} E\left(\frac{A^{2}}{1+A^{2}}\right)-\frac{1}{\sqrt{1+A^{2}}} K\left(\frac{A^{2}}{1+A^{2}}\right)\right)\right] \\
b_{3}=\frac{4 \lambda \omega_{0}^{2}}{3 \pi A^{3} \sqrt{1+A^{2}}}\left[\left(8+9 A^{2}+A^{4}\right) E\left(\frac{A^{2}}{1+A^{2}}\right)-\left(8+5 A^{2}\right) K\left(\frac{A^{2}}{1+A^{2}}\right)\right]
\end{gathered}
$$

where $K(m)$ and $E(m)$ are the complete elliptic integrals of the first and the second kind, respectively, and $E(m)$ is defined as follows

$$
E(m)=\int_{0}^{\pi / 2} \sqrt{1-m \sin ^{2} \theta} \mathrm{d} \theta
$$

In Figure 3 we compare the nonlinear function $f(x) / \omega_{0}^{2}=x-\frac{x}{\sqrt{1+x^{2}}}($ for $\lambda=1$ and $A=1)$ with its cubic approximation obtained using the Chebyshev series expansion, $f_{C h}(x) / \omega_{0}^{2}=0.0494171 x+0.250431 x^{3}$ (for $\lambda=1$ and $\left.A=1\right)$.

The exact frequency can be written as follows $[1,17]$

$$
\omega_{e}(A)=\frac{\pi}{2} \omega_{0}\left[\int_{0}^{1} \frac{A \mathrm{~d} u}{\sqrt{A^{2}\left(1-u^{2}\right)-2 \lambda\left(\sqrt{1+A^{2}}-\sqrt{1+A^{2} u^{2}}\right)}}\right]^{-1}
$$

which must be solved numerically for each value of $A$. As an example we only consider $\lambda=1$. In this situation, and for small values of the amplitude $A$, it is possible to take into account the following approximation 


$$
\begin{aligned}
\omega_{e}(A) & \approx \frac{\Gamma^{2}(3 / 4)}{\sqrt{2 \pi}} \omega_{0} A-\frac{\pi^{3 / 2}\left[3 \Gamma^{2}(3 / 4)+4 \Gamma^{2}(5 / 4)\right]}{256 \sqrt{2} \Gamma^{2}(5 / 4)} \omega_{0} A^{3}+\ldots \\
& \approx 0.59907 \omega_{0} A-0.177537 \omega_{0} A^{3}+\ldots
\end{aligned}
$$

For small values of $A$ it is also possible to do a power-series expansion of the approximate angular frequency $\omega_{C h}(A)$ and we obtain

$$
\begin{aligned}
\omega_{C h}(A) & \approx \frac{\Gamma^{2}(3 / 4)}{\sqrt{2 \pi}} \omega_{0} A-\frac{15 \Gamma^{2}(3 / 4)\left[12 \Gamma^{2}(5 / 4)-\Gamma^{2}(3 / 4)\right]}{512 \sqrt{2 \pi} \Gamma^{2}(5 / 4)} \omega_{0} A^{3}+\ldots \\
& \approx 0.59907 \omega_{0} A-0.178531 \omega_{0} A^{3}+\ldots
\end{aligned}
$$

where $\Gamma(q)$ is the Euler gamma function. These power-series expansion is done using MATHEMATICA. We can easily verify that

$$
\lim _{A \rightarrow \infty} \frac{\omega_{C h}(A)}{\omega_{e}(A)}=1, \quad \quad \lim _{A \rightarrow 0} \frac{\omega_{C h}(A)}{\omega_{e}(A)}=1
$$

which means that the relative error of the angular frequency tends to 0 when $A$ tends to zero and to infinity. In Figure 4 we have plotted the relative error of the approximate frequency as a function of $A$ for $\lambda=1$. From Eq. (43) and Figure 4, we can conclude that $\omega_{C h}(A)$ in Eqs. (21), (33) and (34) gives excellent approximate frequencies for small as well large values of oscillation amplitude $A$.

\section{4.- The dynamically shifted oscillator}

The nonlinear differential equation for this case is [22]

$$
\frac{\mathrm{d}^{2} x}{\mathrm{~d} t^{2}}+\omega_{0}^{2}\left[x+x_{0} \operatorname{sgn}(x)\right]=0, \quad \omega_{0}=\sqrt{\frac{k}{m}}
$$


with initial conditions given in Eq. (2). Parameter $x_{0}$ is the dynamic shift and it can be positive or negative, but in the last situation $A>2\left|x_{0}\right|[8,18]$.

Using the Chebyshev series expansion we obtain

$$
b_{1}=\left(A+\frac{4 x_{0}}{\pi}\right) \omega_{0}^{2}, \quad b_{3}=-\frac{4 x_{0}}{3 \pi} \omega_{0}^{2}
$$

and the approximate frequency is given as

$$
\omega_{C h}(A)=\frac{\pi \sqrt{1+\frac{8 x_{0}}{3 \pi A}}}{2 K\left(-\frac{8 x_{0}}{8 x_{0}+3 \pi A}\right)}
$$

In Figure 5 we compare the nonlinear function $f(x) / \omega_{0}^{2}=x+x_{0} \operatorname{sgn}(x)$ with its cubic approximation obtained using the Chebyshev series expansion, $f_{C h}(x) / \omega_{0}^{2}=\left(1+\frac{8 x_{0}}{\pi A}\right) x-\frac{16 x_{0}}{3 \pi A^{3}} x^{3}$.

The exact frequency $\omega_{e}(A)$ for this oscillator is given as follows [8,18,19]

$$
\omega_{e}(A)=\omega_{0}\left[1-\frac{2}{\pi} \sin ^{-1}\left(\frac{x_{0}}{A+x_{0}}\right)\right]^{-1}
$$

For small values of the amplitude $A$, it is possible to take into account the following approximation

$$
\omega_{e}(A) \approx \frac{\pi \omega_{0}}{2} \sqrt{\frac{x_{0}}{2 A}}+\ldots=1.11072 \omega_{0} \sqrt{\frac{x_{0}}{A}}+\ldots
$$

For small values of $A$ it is also possible to do a power-series expansion of the approximate angular frequency $\omega_{C h}(A)$ and we obtain 
Beléndez, A.; Álvarez, M. L., Fernández, E., Pascual, I., "Cubication of conservative nonlinear oscillators", European Journal of Physics, Vol. 30, № 5, pp. 973-981 (2009). doi:10.1088/0143-0807/30/5/006

$$
\omega_{C h}(A) \approx \frac{\Gamma(3 / 4) \omega_{0}}{\Gamma(5 / 4)} \sqrt{\frac{2 x_{0}}{3 A}}+\ldots=1.10387 \omega_{0} \sqrt{\frac{x_{0}}{A}}+\ldots
$$

We can easily verify that

$$
\lim _{A \rightarrow \infty} \frac{\omega_{C h}(A)}{\omega_{e}(A)}=1, \quad \quad \lim _{A \rightarrow 0} \frac{\omega_{C h}(A)}{\omega_{e}(A)}=0.993830
$$

which means that the relative error of the angular frequency tends to $0.62 \%$ when $A$ tends to zero.

\section{Conclusions}

A cubication method for conservative nonlinear oscillators based on the Chebyshev series expansion of the restoring force considered previously by Jonckheere [3] and other authors has been analyzed and discussed and an approximate frequency-amplitude relationship has been obtained. In this procedure, instead of approximately solve the original nonlinear differential equation, this is replaced with a Duffing equation which is exactly solved. We think this method has some pedagogical value to both graduate as well as undergraduate students. Some examples of nonlinear oscillators have been approximately solved using this scheme and good results have been obtained, not only for small but also for large values of the oscillation amplitude.

\section{Acknowledgment}

This work was supported by the 'Vicerrectorado de Tecnología e Innovación Educativa' of the University of Alicante, Spain (GITE-09006-UA). 
Beléndez, A.; Álvarez, M. L., Fernández, E., Pascual, I., "Cubication of conservative nonlinear oscillators”, European Journal of Physics, Vol. 30, N 5, pp. 973-981 (2009). doi:10.1088/0143-0807/30/5/006

\section{References}

[1] Beléndez A, Álvarez M L, Fernández E and Pascual I 2009 Linearization of conservative nonlinear oscillators Eur. J. Phys. 30 259-70

[2] Denman H H 1969 An approximate equivalent linearization technique for nonlinear oscillations J. Appl. Mech. 36 358-60

[3] Jonckheere R E 1971 Determination of the period of nonlinear oscillations by means of Chebyshev polynomials ZAMM-Zeitschrift fur Angewandte Mathematik und Mechanik 55 $389-93$

[4] Bravo Yuste S 1992 "Cubication" of non-linear oscillators using the principle of harmonic balance Int. J. Non-Linear Mech. 27 347-56

[5] Marion J B and Thornton S T 1995 Classical Dynamics of Particles and Systems (New York: Saunders)

[6] Mickens R E 1996 Oscillations in Planar Dynamics Systems (Singapore: World Scientific).

[7] Amore P, Raya A and Fernández F M 2005 Alternative perturbation approaches in classical mechanics Eur. J. Phys. 26, 1057-63

[8] Mohazzabi P 2004 Theory and examples of intrinsically nonlinear oscillators Am. J. Phys. $72492-8$

[9] Abramowitz M. and Stegun I A (Eds.) 1972 "Orthogonal Polynomials", Ch. 22 in Handbook of Mathematical functions with Formulas, Grapas and Mathematical Tables, 9th printing. New York: Dover, pp. 771-802.

[10] Weisstein E W Chebyshev Polynomial of the First Kind. From MathWorld-A Wolfram web resource. http://mathworld.wolfram.com/ChebyshevPolynomialoftheFirstKind.html

[11] Parwani R R 2004 An approximate expression for the large angle period of a simple pendulum Eur. J. Phys. 25, 37-49 
Beléndez, A.; Álvarez, M. L., Fernández, E., Pascual, I., "Cubication of conservative nonlinear oscillators", European Journal of Physics, Vol. 30, N 5, pp. 973-981 (2009). doi:10.1088/0143-0807/30/5/006

[12] Beléndez A, Hernández A, Márquez A, Beléndez T and Neipp C 2006 Analytical approximations for the period of a simple pendulum Eur. J. Phys. 27 539-51

[13] Lima F M S and Arun P 2006 An accurate formula for the period of a simple pendulum oscillating beyond the small angle regime Am. J. Phys. 74 892-5

[14] Amore P, Valdovinos M C, Orneles G and Barajas S Z 2007 The nonlinear pendulum: formulae for the large amplitude period Rev. Mex. Fis. E 53 106-111

[15] Beléndez A, Hernández A, Beléndez T, Márquez A and Neipp C 2007 An improved 'heuristic' approximation for the period of a nonlinear pendulum: linear analysis of a nonlinear problem Int. J. Non-linear Sci. Numer. Simulat. 8 329-34

[16] Lima F M S 2008 Simple 'log formulae' for pendulum motion valid for any amplitude Eur. J. Phys. 29 1091-8

[17] Beléndez A, Hernández A, Beléndez T, Álvarez M L, Gallego S, Ortuño M and Neipp, C 2007 Application of the harmonic balance method to a nonlinear oscillator typified by a mass attached to a stretched wire J. Sound Vib. 302 1018-29

[18] Hartmann W M 1986 The dynamically shifted oscillator Am. J. Phys. 54 28-31

[19] Beléndez A, Rodes J J, Fuentes R, García C, Yebra M S and Pascual I 2009 Linearized harmonic balancing approach for accurate solutions to the dynamically shifted oscillar Int. J. Non-Linear Sci. Numer. Simulat. 10 509-22 
Beléndez, A.; Álvarez, M. L., Fernández, E., Pascual, I., "Cubication of conservative nonlinear oscillators", European Journal of Physics, Vol. 30, № 5, pp. 973-981 (2009). doi:10.1088/0143-0807/30/5/006

\section{FIGURE CAPTIONS}

Figure 1.- Comparison between the nonlinear function $f(x) / \omega_{0}^{2}$ with their cubication approximations obtained using the Taylor series expansion, $f_{T}(x) / \omega_{0}^{2}$, and the Chebyshev series expansion, $f_{C h}(x) / \omega_{0}^{2}$, for the simple pendulum with $A=120^{\circ}$.

Figure 2.- Comparison between the nonlinear function $f(x) / \omega_{0}^{2}$ with its cubication approximation obtained using the Chebyshev series expansion, $f_{C h}(x) / \omega_{0}^{2}$, for the antisymmetric, constant force oscillator with $A=1$.

Figure 3.- Comparison between the nonlinear function $f(x) / \omega_{0}^{2}$ with its cubication approximation obtained using the Chebyshev series expansion, $f_{C h}(x) / \omega_{0}^{2}$, for a mass attached to two stretched elastic springs with $\lambda=1$ and $A=1$.

Figure 4.- Percentage of error for the approximate frequency obtained using a cubication procedure based on the Chebyshev series expansion for a mass attached to two stretched elastic springs with $\lambda=1$.

Figure 5.- Comparison between the nonlinear function $f(x) / \omega_{0}^{2}$ with its cubication approximation obtained using the Chebyshev series expansion, $f_{C h}(x) / \omega_{0}^{2}$, for the dynamically shifted oscillator with $x_{0}=0.4$ and $A=1$. 
Beléndez, A.; Álvarez, M. L., Fernández, E., Pascual, I., "Cubication of conservative nonlinear oscillators", European Journal of Physics, Vol. 30, N 5, pp. 973-981 (2009). doi:10.1088/0143-0807/30/5/006

\section{FIGURE 1}

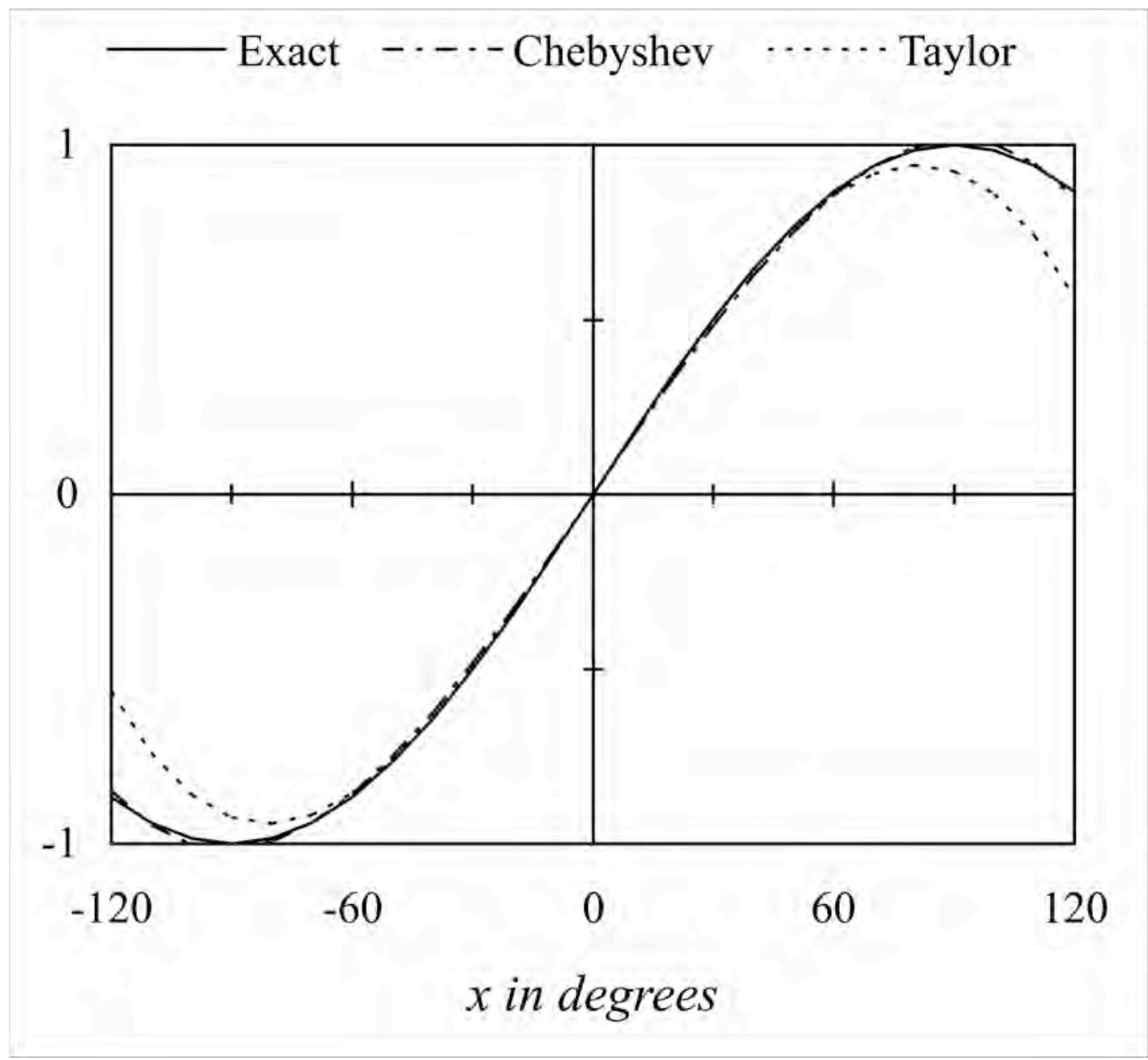


Beléndez, A.; Álvarez, M. L., Fernández, E., Pascual, I., "Cubication of conservative nonlinear oscillators", European Journal of Physics, Vol. 30, № 5, pp. 973-981 (2009). doi:10.1088/0143-0807/30/5/006

\section{FIGURE 2}

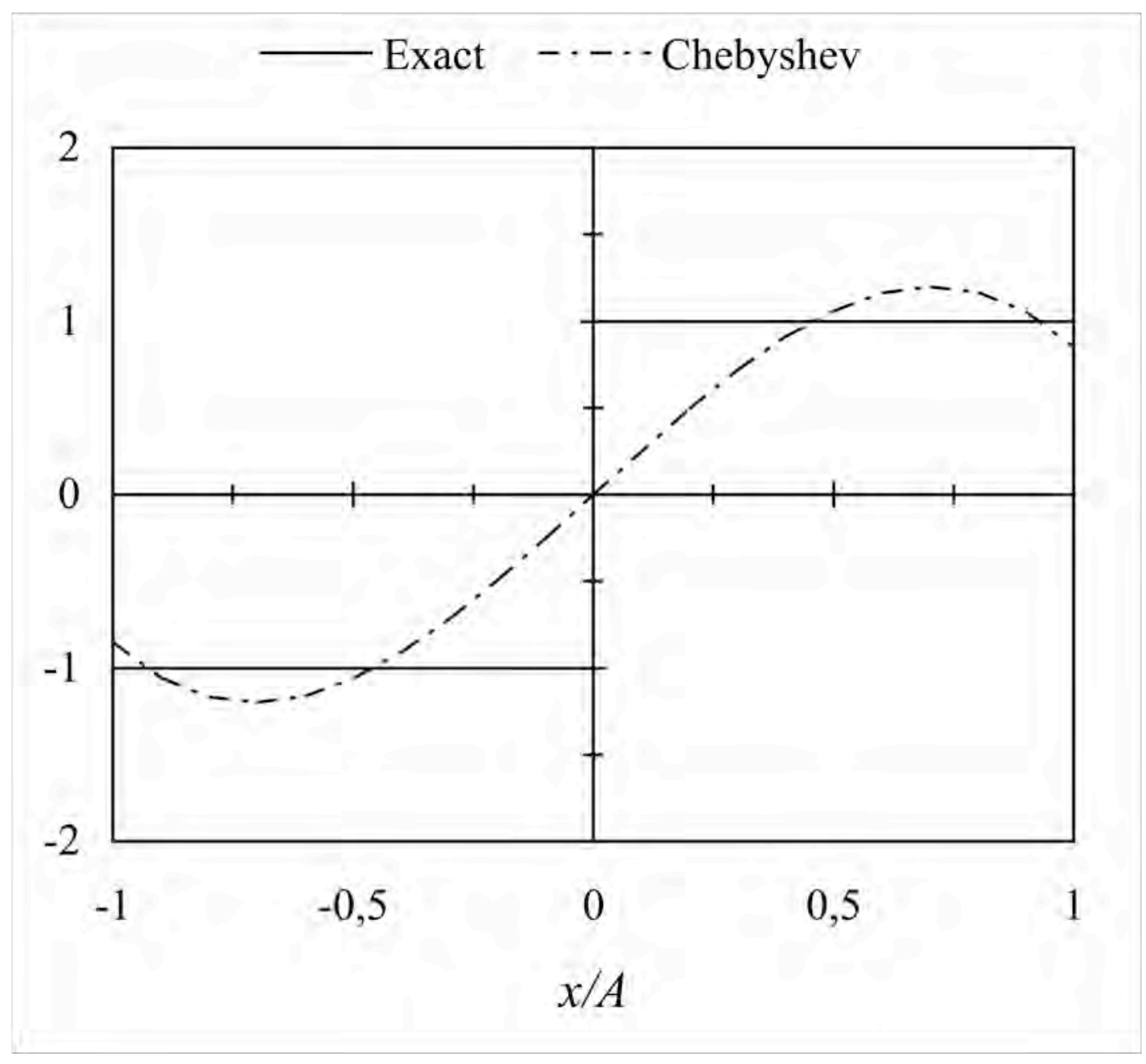


Beléndez, A.; Álvarez, M. L., Fernández, E., Pascual, I., "Cubication of conservative nonlinear oscillators”, European Journal of Physics, Vol. 30, № 5, pp. 973-981 (2009). doi:10.1088/0143-0807/30/5/006

\section{FIGURE 3}

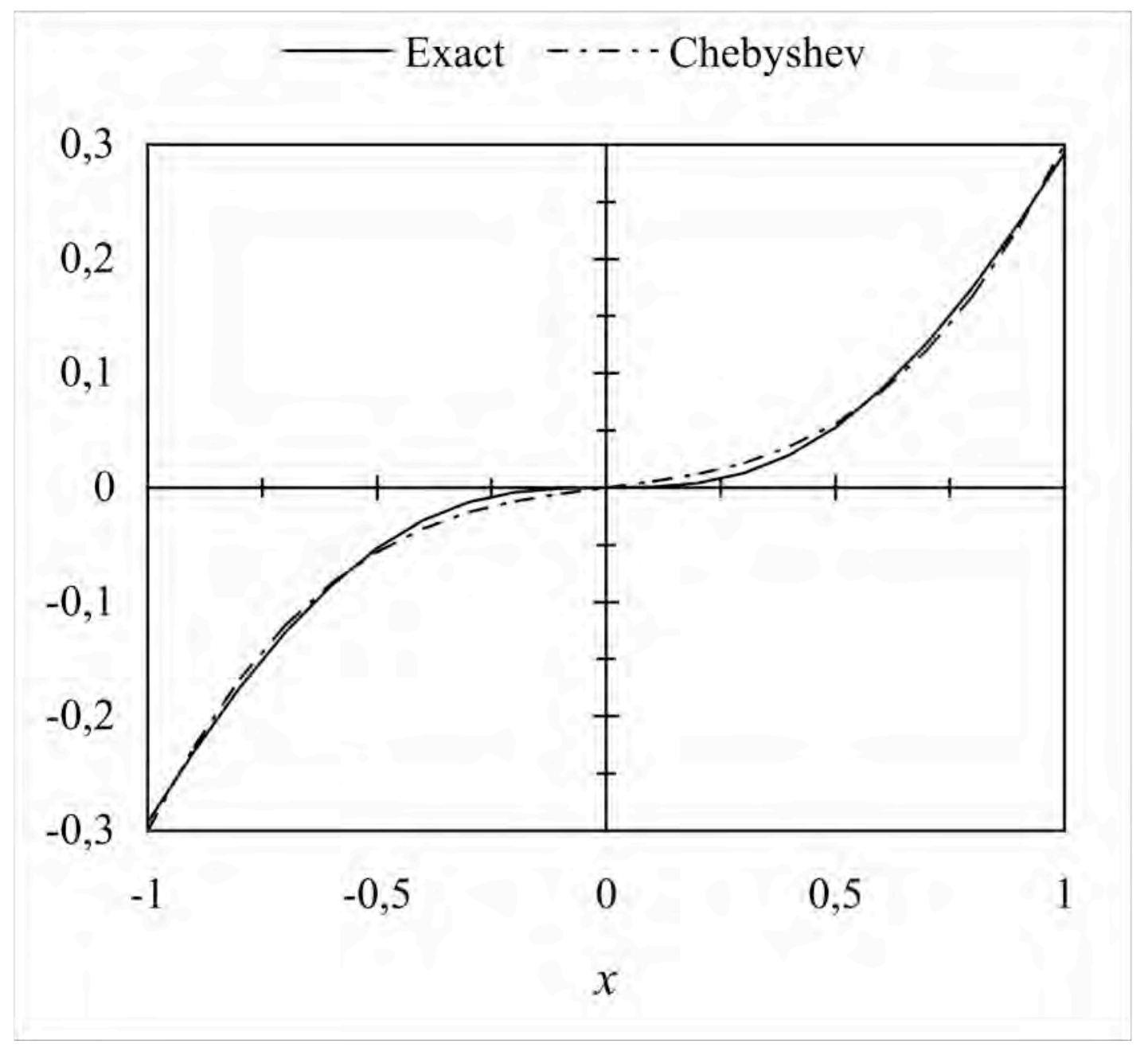


Beléndez, A.; Álvarez, M. L., Fernández, E., Pascual, I., "Cubication of conservative nonlinear oscillators", European Journal of Physics, Vol. 30, № 5, pp. 973-981 (2009). doi:10.1088/0143-0807/30/5/006

\section{FIGURE 4}

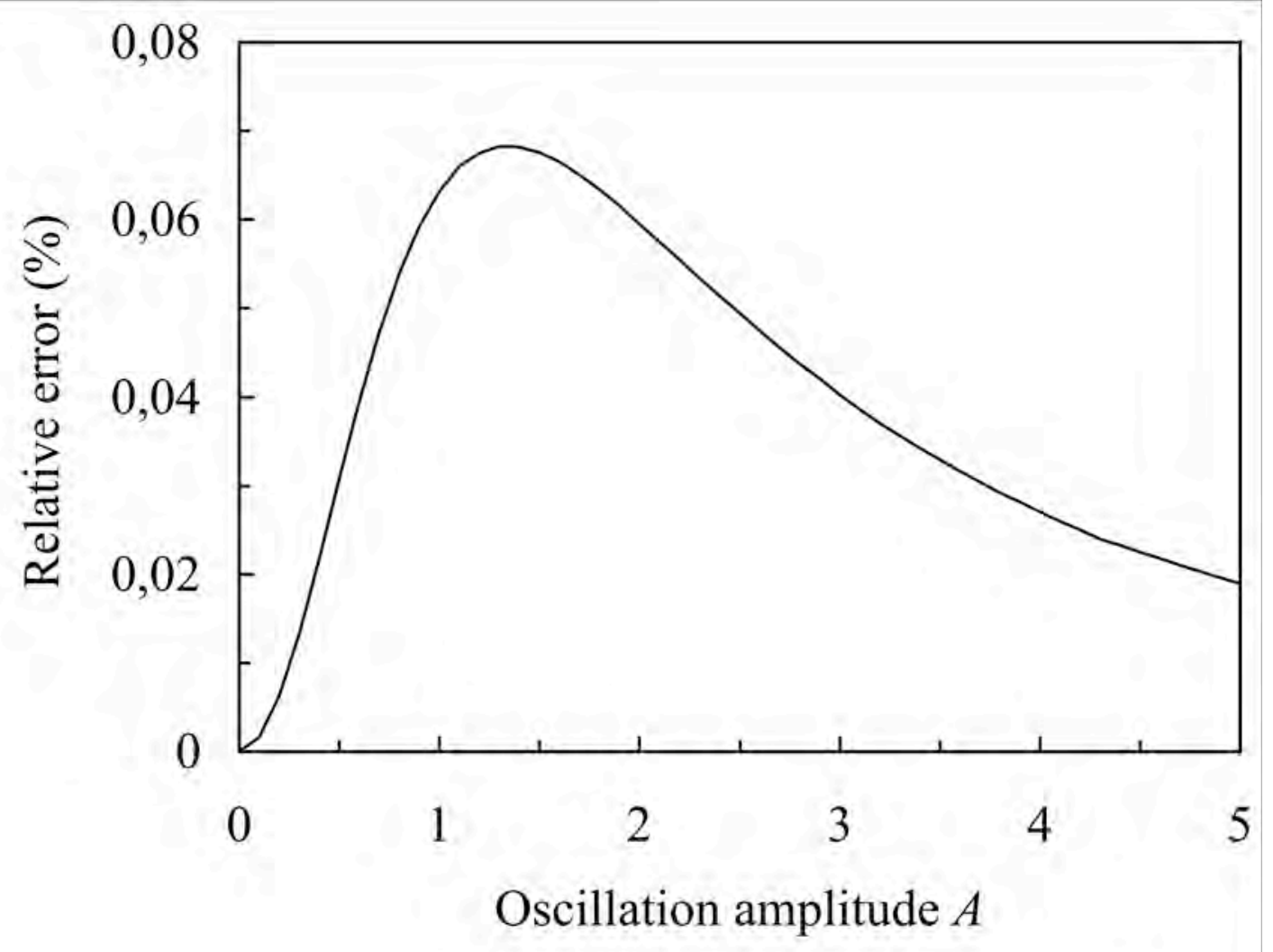


Beléndez, A.; Álvarez, M. L., Fernández, E., Pascual, I., "Cubication of conservative nonlinear oscillators”, European Journal of Physics, Vol. 30, № 5, pp. 973-981 (2009). doi:10.1088/0143-0807/30/5/006

\section{FIGURE 5}

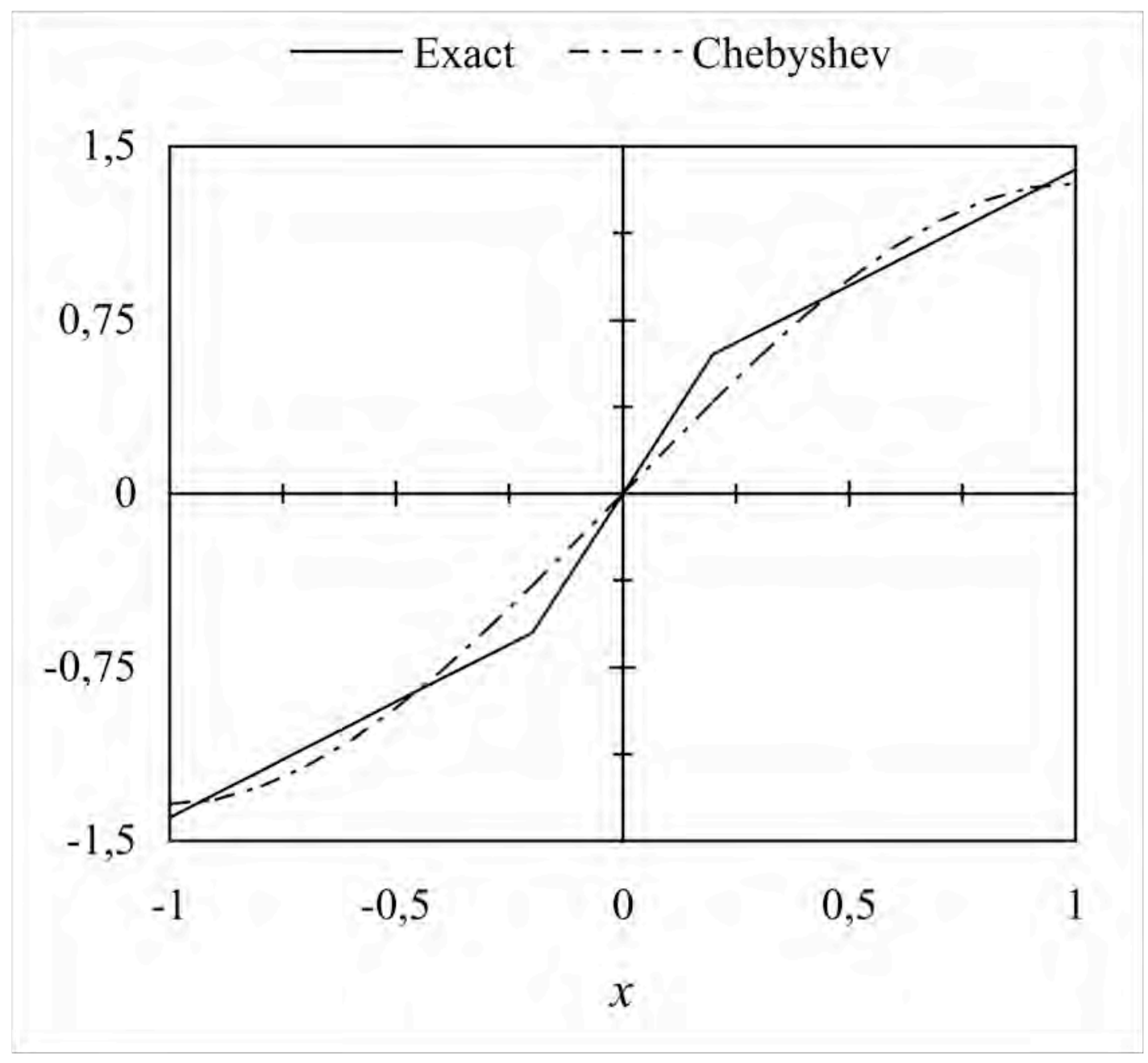

